\title{
$5-2020$
}

\section{Neurological manifestations of COVID-19}

\author{
Dureshahwar Kanwar \\ Aga Khan University, dureshahwar.kanwar@aku.edu \\ Abdul Mannan Baig \\ Aga Khan University, abdul.mannan@aku.edu \\ Mohammad Wasay \\ Aga Khan University, mohammad.wasay@aku.edu
}

Follow this and additional works at: https://ecommons.aku.edu/pakistan_fhs_mc_med_neurol

Part of the Life Sciences Commons, Neurology Commons, and the Virus Diseases Commons

\section{Recommended Citation}

Kanwar, D., Baig, A. M., Wasay, M. (2020). Neurological manifestations of COVID-19. JPMA. The Journal of the Pakistan Medical Association, 70(5(Suppl 3)), S101-S103.

Available at: https://ecommons.aku.edu/pakistan_fhs_mc_med_neurol/214 


\section{Neurological manifestations of COVID-19}

Dureshahwar Kanwar, ${ }^{1}$ Abdul Mannan Baig,, ${ }^{2}$ Mohammad Wasay ${ }^{3}$

\begin{abstract}
Coronavirus disease (COVID-19) caused by severe acute respiratory syndrome coronavirus 2 (SARS CoV-2) has turned out to be a formidable pandemic. Upcoming evidence from confirmed cases of COVID-19 suggests an anticipated incursion of patients with neurological manifestations in the weeks to come. An expression of the angiotensin-converting enzyme 2 (ACE 2), the cellular receptor for SARS-CoV-2 over the glial cells and neurons have made the brain a potential target. Neurotoxicity may occur as a result of direct, indirect and post-infectious complications. Attention to neurological deficits in COVID19 is fundamental to ensure appropriate, timely, beneficial management of the affected patients. Most common neurological manifestations seen include dizziness, headache, impaired consciousness, acute cerebrovascular disease, ataxia, and seizures. Anosmia and ageusia have recently been hinted as significant early symptoms in COVID-19. As cases with neurological deficits in COVID-19 emerge, the overall prognosis is yet unknown.
\end{abstract}

Keywords: Coronavirus, SARS-CoV-2, COVID-19, ACE 2, Neurotoxicity, Brain, Seizures.

\section{DOI: https://doi.org/10.5455/JPMA.20}

\section{Introduction}

Neurological symptoms of coronavirus disease (COVID19) can be minimal at presentation but these can cause monumental damage as silent contagious sources. With the recent outbreak, there is a compelling need to understand the potential of the severe acute respiratory syndrome coronavirus 2 (SARS-CoV-2) to cause neurological illness for proper diagnosis and therapeutic decision making.

The SARS-CoV-2 particle resembles a medieval European royal crown hence the name. The viral particle is enclosed by an envelope, and protein spikes from its membrane surface are responsible for interaction with host cell receptor and resultant virulence and infectivity.

${ }^{1-3}$ Department of Medicine and Biological and 2Biomedical Science, Aga Khan University, Karachi.

Correspondence: Mohammad Wasay. Email: Mohammad.wasay@aku.edu
Angiotensin-converting enzyme 2 (ACE 2) is the cellular receptor for the new SARS-CoV-2 present on the brain and glial tissue and thus this makes the central nervous system (CNS) a potential target. ${ }^{1}$ There may be direct infiltration of the CNS through the nasal epithelium and entrance into the olfactory pathway with the subsequent retrograde trans-synaptic spread. However, secondary neurologic manifestations of COVID-19 are thought to be the result of widespread dysregulation of homeostasis and cytokine production with pulmonary, renal, hepatic, and cardiovascular injury. ${ }^{2}$

Neurological symptoms fall into three main categories: CNS, peripheral nervous symptoms (PNS) and skeletal muscular symptoms. ${ }^{3}$ These clinical features may be the presenting complaints or as more commonly seen arise during the more frequently seen respiratory and febrile illness. CNS manifestations most commonly seen include dizziness, headache, impaired consciousness, acute cerebrovascular disease, ataxia, and seizures. PNS manifestations included taste impairment, smell impairment and nerve pain. Skeletal muscular injury manifestations include myalgias and rhabdomyolysis ${ }^{3}$ (Table-1). These are likely due to significantly elevated pro-inflammatory cytokines in the serum and subsequent downregulation of anti-inflammatory cytokines. Besides coronavirus specific antibodies have been shown to activate macrophages resulting in their migration into the CNS and ultimately cause demyelination. ${ }^{4}$

The American Academy of Otolaryngology recently

Table-1: Neurological Clinical Abnormalities with coronavirus disease (COVID-19).

1. Central Nervous system:
a. Dizziness
b. Headache
c. Impaired consciousness
d.A cute cerebrovascular disease
e. Ataxia
f. Seizures
2. Peripheral Nervous System:
a. Anosmia
b. Ageusia
c. Neuralgia
3. Skeletal Muscle:
a. Myalgia


proposed that anosmia, hyposmia, and dysgeusia should be added to the list of COVID-19 screening symptoms and urged precautionary isolation for individuals with these symptoms, even in the absence of respiratory disease. ${ }^{5}$

In the largest study to date on 214 patients from Wuhan, China, overall, 78 patients (36.4\%) had neurologic manifestations. The mean age of these patients was 52.7 years. Of these patients, 83 (38.8\%) had at least 1 of the following underlying disorders: hypertension (51 [23.8\%]), diabetes (30 [14.0\%]), cardiac or cerebrovascular disease (15 [7.0\%]), and malignancy (13 [6.1\%]). ${ }^{3} \mathrm{~A}$ total of 126 patients $(58.9 \%)$ had non-severe infection and 88 patients $(41.1 \%)$ had severe infection according to their respiratory status. Patients with severe infection were more likely to develop neurologic manifestations, especially acute cerebrovascular disease [5.7\%]; conscious disturbance [14.8\%] and skeletal muscle injury [19.3\%]. ${ }^{3}$ Another recent report of 99 COVID-19 patients in Wuhan also described neurological complications with confusion (9\%) and headache (8\%) being most commonly seen. ${ }^{6}$ Loss of smell and taste may precede respiratory symptoms and suggest early neurological involvement. Respiratory failure in the absence of severe lung involvement may suggest neurogenic respiratory failure. ${ }^{7}$

Most neurologic manifestations occur early in the illness (median time, 1-2 days). Of 6 patients with acute cerebrovascular disease, 2 arrived at the emergency department owing to the sudden onset of hemiplegia but without any typical symptoms of fever, cough, anorexia, and diarrhoea of COVID-19. Their lung lesions were found by an emergent lung CT and were diagnosed as having COVID-19 by a positive SARS-CoV-2 nucleic acid detection in the later stage. ${ }^{3}$

Isolated new case reports of meningitis/encephalitis, ${ }^{8}$ haemorrhagic necrotizing encephalopathies, ${ }^{9}$ GuillainBarre syndrome, ${ }^{10}$ with COVID-19 are emerging every day from all around the world. However, the neuroinvasive nature of the human coronavirus and an association with demyelination including multiple sclerosis ${ }^{11}$ and acute disseminated encephalomyelitis has long been known. ${ }^{12}$

Laboratory findings in patients with COVID -19 include high white blood cell counts, lower lymphocyte count, and increased C-reactive protein. Severe infection have corresponding higher D-dimer levels, increased lactate dehydrogenase, alanine aminotransferase, and aspartate aminotransferase levels, with elevated blood urea nitrogen, creatinine levels, and creatinine kinase (CPK) levels ${ }^{3}$ (Table-2).

Directed neurological workup depending upon the
Table-2: Laboratory findings in coronavirus disease (COVID-19).

\begin{tabular}{ll}
\hline Non Severe Infection & Severe Infections \\
\hline Dearanged white blood cell count & Increased D dimer \\
Raised neutrophil count & Raised Creatine kinase \\
Lower lymphocyte count & Increased Lactate dehydrogenase \\
Low Platelet count & Raised Alanine aminotransferase \\
Increased C reactive protein & Raised Aspartate aminotransferase \\
& Raised Blood Urea Nitrogen \\
& Raised Creatinine \\
\hline
\end{tabular}

clinical concern is required. A non-contrast CT Head to exclude stroke in patients with possible or confirmed COVID 19 should be reserved for times when actionable information is likely to be obtained, or when a significant change in the neurological examination is seen. A CT angiogram of the head and neck may be required in cases of subarachnoid hemorrhage. Additional studies including MRI, echocardiograms and doppler should be considered only if there is a high likelihood of changing management as contamination issues are present. All electroencephalogram (EEG) orders must be discussed with EEG service attending and cases of seizures or possibility of non-convulsive seizures (seen in up to $10 \%$ of critically-ill patients) should ideally be performed with disposable EEG leads. A cerebrospinal fluid (CSF) examination may be required at the discretion of the neurology consultant to exclude possible causes of impaired consciousness. A lumbar puncture to look for SARS-CoV-2 nucleic acid by using PCR is recommended in highly suspicious cases.

An over-emphasis on the fact that nerve conduction studies and needle electromyography are usually within normal limits in the first 1-2 weeks of diseases of the muscle and nerves cannot be made. No unnecessary procedures should be performed as the machines may serve as fomites and this needs close consideration.

Standard management of stroke with suspected COVID19 requires certain considerations. Patients needing tissue plasminogen activator (tPA) during the one-hour infusion may be monitored every 15 minutes by one member of the stroke team in the patient's room to conserve personal protective equipment (PPE) and limit the potential for accidental exposure. In cases of mechanical thrombectomy, groin checks can be done by team members entering for other reasons every 4 hours after the initial monitoring in the intervention radiology suite.

Limiting exposure to potentially infected patients of medical personnel and possible tele-neurology 
consultations when possible cannot be overstated. Antiepileptic drugs that do not cause liver or renal damage should be considered for control of seizures and valproate should also be avoided in acute headache at this time in the context of its known hepatotoxicity.

Also, the putative role of ACE2 in the pathophysiology of COVID-19 has recently fueled concerns regarding the use of non- steroidal anti-inflammatory drugs (NSAIDs) such as ibuprofen, commonly used for headache. These are known to induce up-regulation of ACE2 and, in theory, may facilitate infection with COVID-19.13

The role of high dose intravenous immunoglobulins (IVlg) in a recent case series of patients with COVID-19, all of whom were successfully treated at the early stage of clinical deterioration has been published with the resultant initiation of a randomized controlled trial. ${ }^{14}$ This may lead us to rethink the equal efficacy that has been long established when treating patients with muscle or nerve involvement with plasma exchange versus IVIg.

Practicing neurologists amid these critical times have considerable unanswered queries in their minds especially in underdeveloped countries. Do isolated neurological symptoms warrant a COVID-19 testing there and then before being a silent contagious source? Do we take sufficient steps in educating our patients who are on long-term use of immunosuppressants for illnesses like myasthenia gravis, multiple sclerosis, and sarcoidosis, and are now at a higher risk for COVID -19? Do Asians have an additional risk for strokes in these times because of the high prevalence of intracranial atherosclerosis here? Do larger pharmaceuticals need to change direction in the development of newer drugs and emphasise more on antiviral agents considering that only a handful are available? Is the COVID-19 here to stay? Will it become just become another herpes, dengue, chikungunya infectioncausing neurological manifestations that we have been commonly seeing over the past several years?

\section{Conclusion}

A cross-disciplinary unified approach is required in this herculean endeavour by mankind to control this outbreak as it still unfolds. Is this just a cursory glimpse of more formidable events to be revealed in the future? Only time will tell. Healthcare providers should keep a close watch for isolated neurological symptoms at presentation when evaluating patients for COVID-19 to assure both early appropriate management and control of disease. Research for new antiviral agents and the development of a possible vaccine is paramount and the time for action is now. This is the only way to assure containment of this suffering of humanity and its continued existence.

\section{References}

1. Yan R, Zhang Y, Li Y, Xia L, Guo Y, Zhou Q. Structural basis for the recognition of SARS-CoV-2 by full-length human ACE2. Science 2020;367:1444-8. doi: 10.1126/science.abb2762.

2. Baig AM, Khaleeq A, Ali U, Syeda H. Evidence of the COVID-19 virus targeting the cns: tissue distribution, host-virus interaction, and proposed neurotropic mechanisms. ACS Chem Neurosci 2020;11:995-8. doi: 10.1021/acschemneuro.0c00122.

3. Mao L, Jin H, Wang M, Hu Y, Chen S, He Q, et al. Neurologic manifestations of hospitalized patients with coronavirus disease 2019 in Wuhan, China. JAMA Neurol 2020:e201127. doi: 10.1001/jamaneurol.2020.1127.

4. Kim TS, Perlman S. Viral expression of CCL2 is sufficient to induce demyelination in RAG1-/- mice infected with a neurotropic coronavirus. J Virol 2005;79:7113-20. doi: 10.1128/JVI.79.11.71137120.2005

5. Giacomelli A, Pezzati L, Conti F, Bernacchia D, Siano M, Oreni L, et al. Self-reported olfactory and taste disorders in SARS-CoV-2 patients: a cross-sectional study. Clin Infect Dis 2020:ciaa330. doi: 10.1093/cid/ciaa330.

6. Chen N, Zhou M, Dong X, Qu J, Gong F, Han Y, et al. Epidemiological and clinical characteristics of 99 cases of 2019 novel coronavirus pneumonia in Wuhan, China: a descriptive study. Lancet 2020;395:507-13. doi: 10.1016/S01406736(20)30211-7.

7. Baig AM, Wasay M. COVID-19 infection; Loss of taste, smell and neurogenic respiratory failure. Pakistan Journal of Neurological Sciences (in press)

8. Moriguchi T, Harii N, Goto J, Harada D, Sugawara H, Takamino J, et al. A first case of meningitis/encephalitis associated with SARSCoronavirus-2. Int J Infect Dis 2020;94:55-8. doi: 10.1016/j.jijid.2020.03.062.

9. Poyiadji N, Shahin G, Noujaim D, Stone M, Patel S, Griffith B. COVID-19-associated acute hemorrhagic necrotizing encephalopathy: CT and MRI features. Radiology 2020:201187. doi: 10.1148/radiol.2020201187

10. Zhao H, Shen D, Zhou H, Liu J, Chen S. Guillain-Barré syndrome associated with SARS-CoV-2 infection: causality or coincidence? Lancet Neurol. 2020 May;19(5):383-384. doi: 10.1016/S14744422(20)30109-5.

11. Cristallo A, Gambaro F, Biamonti G, Ferrante P, Battaglia M, Cereda PM. Human coronavirus polyadenylated RNA sequences in cerebrospinal fluid from multiple sclerosis patients. New Microbiol 1997;20:105-14.

12. Yeh EA, Collins A, Cohen ME, Duffner PK, Faden H. Detection of coronavirus in the central nervous system of a child with acute disseminated encephalomyelitis. Pediatrics 2004;113(1 Pt 1):e736. doi: 10.1542/peds.113.1.e73.

13. Fang L, Karakiulakis G, Roth M. Are patients with hypertension and diabetes mellitus at increased risk for COVID-19 infection? Lancet Respir Med. 2020 Apr;8(4):e21. doi: 10.1016/S22132600(20)30116-8.

14. Cao W, Liu X, Bai T, Fan H, Hong K, Song H, Han Y, Lin L, Ruan L, Li T. High-Dose Intravenous Immunoglobulin as a Therapeutic Option for Deteriorating Patients With Coronavirus Disease 2019. Open Forum Infect Dis. 2020 Mar 21;7(3):ofaa102. doi: 10.1093/ofid/ofaa102. PMID: 32258207; PMCID: PMC7111600. 